\title{
海洋水下立体观测技术装备发展研究
}

\author{
马荇 ${ }^{1}$, 赵修涛 $^{2}$, 柳存根 $^{1}$ \\ (1. 上海交通大学船舶海洋与建筑工程学院, 上海 200240；2. 哈尔滨工程大学科学技术研究院, 哈尔滨 150001)
}

\begin{abstract}
摘要: 建立水下立体观测网来获得科学、实时、全面的数据, 是未来认识、开发、利用海洋的重要方向。本文分析了发展海 洋水下立体观测技术装备的需求和必要性, 对比了国内外相关装备的发展现状, 进一步剖析我国领域发展面临的问题, 研判 作为海洋水下观测关键环节的传感器技术进展点。研究认为, 我国海洋观测平台技术有了很大进步, 但在海洋关键传感器、 高精度传感器方面依然落后于世界先进水平; 海洋观测的大数据与实际需求之间有所脱节, 海洋传感器缺乏改进平台支撑。 研究提出了支持海洋关键传感器研究成果高效转化、统筹管理国家海洋水下立体观测技术装备、建立海上仪器装备国家公共 试验平台等对策建议, 以期为相关领域中长期发展提供方向参照。
\end{abstract}

关键词: 海洋观测; 海底观测网; 水下移动观测平台; 水下传感器

中图分类号: P715 文献标识码: A

\section{Development of Marine Equipment for Underwater Stereoscopic Observation}

\author{
Ma Rui $^{1}$, Zhao Xiutao ${ }^{2}$, Liu Cungen ${ }^{1}$ \\ (1. School of Naval Architecture, Ocean \& Civil Engineering, Shanghai Jiao Tong University, Shanghai 200240, China; \\ 2. College of Science and Technology, Harbin Engineering University, Harbin 150001, China)
}

\begin{abstract}
Establishing an underwater stereoscopic observation network to obtain scientific, real-time, and comprehensive data is an important direction for understanding and exploiting the ocean in the future. This study first analyzes the need and necessity of developing the underwater stereoscopic observation equipment, then introduces the development status of the equipment in China and abroad, further analyzes the problems faced by China's field development, and presents the key breakthrough points of marine sensors which serve as the key link for underwater observation. China has made great progress in technologies regarding marine observation platforms, but it still lags behind the world advanced level in key marine sensors and high-precision sensors. The big data obtained from ocean observation does not match the actual demand, and the ocean sensors lack the support from an improved platform. To guide the long-term development of related fields, we suggest that China should support the efficient transformation of research results of key marine sensors, coordinate the management of the marine equipment for underwater stereoscopic observation, and establish a national public test platform for offshore instruments and equipment.
\end{abstract}

Keywords: ocean observation; sea floor observatory network; underwater vehicle; underwater sensor

收稿日期 : 2020-10-07; 修回日期 : 2020-11-11

通讯作者: 柳存根, 上海交通大学船舶海洋与建筑工程学院教授, 研究方向为船舶设计、船舶智能化、海洋装备战略;

E-mail:cgliu@sjtu.edu.cn

资助项目：中国工程院咨询项目“海洋装备发展战略研究” (2020-ZD-02)

本刊网址 : www.engineering.org.cn/ch/journal/sscae 


\section{一、前言}

海洋观测依赖于装备的技术进步：早期人类 认识海洋主要是从船上或者岸上; 20 世纪遥感技术 的出现使得从空间获取海洋动态性信息成为可能; 进入 21 世纪, 从水下和海底观测海洋成为探索热 点。作为时空耦合的复杂过程, 海洋过程的观察 需要协同使用移动和固定的观测平台。海洋水下 立体观测系统指基于有线或无线方式，借助固定 或移动的平台, 对海洋进行实时观测的网络 [1]。 海洋观测技术装备是观测系统的基础, 是维护海 洋安全、防控海洋灾害、促进海洋科学进步的重 要支撑。

20 世纪 60 年代起, 国际上许多海洋科学考察、 海洋资源活动开始探索利用各类水下观测技术装 备。例如, 水下遥控装置作为无人水下观测平台, 具有一定的机动性, 可在较大水深中长时间工作, 获得了迅速发展 [2]。我国海洋科学家在 1997 年提 出了从海底观察地球的倡议, 认为海底观测网建设 存在国际竞争, 可能引发国际权益与安全之争, 我 国宜尽早着手、争取主动 [3]; 在分析加拿大海底 科学长期观测网研究进展的基础上, 阐述了海底观 测网的价值 [4]。国家海洋技术中心发表的系列论 文全面介绍了国际海洋水下立体观测系统发展的情 况, 如文献 [5] 介绍了水下自航式海洋观测平台, 分析领域发展历史和现状, 提出我国开展相关研究 的重要性和可能方向; 文献 [6] 分析了国内外水下 观测信息体系的现状与趋势, 提出我国水下观测信 息体系发展建议。

海洋水下立体观测网通常分为三部分：海底观 测网、水下移动观测平台及传感器、水下立体观测 信息系统。本文主要围绕前面两个部分展开研究, 论述发展需求, 在概要梳理国内外领域进展的基础 上总结发展趋势、凝练存在问题、分析关键技术, 据此提出领域发展建议。

\section{二、海洋水下立体观测技术装备的需求分析}

\section{（一）促进海洋科学发展}

海洋科学是基于观测的学科, 新发现、新进步 都离不开海洋观测技术装备。全面提升海洋水下立 体观测装备水平, 是促进我国海洋科学发展的需要,
也是解决海洋科学重大问题的关键。假如将地面与 海面视为地球科学的第一个观测平台、空中视为第 二个观测平台, 则海底是第三个观测平台。海洋水 下立体观测平台将从水面到海底的范围纳入人类的 探测监测视域, 有望从根本上改变人类认识海洋的 途径, 开创海洋科学的新阶段 [3]。

海洋科学方面的重大发现与科学问题, 需要建 立在长期观测的基础上才能获得突破。发展海洋水 下观测技术装备, 为科学家提供了从水面到水下、 从水中到海底地壳深部、全方位、大范围、综合性、 实时性的高精度观测条件; 通过观测, 更好理解 人类活动导致的海洋变化、海洋生命、海底 - 海 水 - 大气之间关联、海底及沉积动力学等海洋科 学问题。

\section{（二）防控海洋灾害}

海洋观测是海洋灾害有效预测预警的信息来 源, 世界上有约 $80 \%$ 的火山喷发、地震发生在海底, 且主要沿着地壳的边界 [3]。水下立体观测技术装 备对海底地震进行监测、评级、定位, 通过数据的 处理、建模、分析, 准确预报台风、海啸等自然灾 害; 监测风暴、藻类勃发、海底喷发、滑坡等各种 突发事件 [5], 降低灾害带来的经济损失, 为沿海 地区的经济社会发展提供防灾减灾保障。

\section{（三）保障海上国防安全}

发展海洋水下立体观测技术装备是海上国防的 必然需求。我国海岸线长约 $18000 \mathrm{~km}$, 海上邻国 有 8 个, 且一些国家与我国存在领土/领海纠纷。 一些国家在第一岛链设置了海底声学监听网络系 统, 对我国海军装备的安全出入构成威胁。为了避 免海洋水下 “门户洞开”、水下空间 “单向透明” 状态, 自主建设水下监测系统、发展水下监测装备 是我国海上国防建设的必然需求。

海洋水下立体观测技术装备具有长期、实时、 不间断、大范围的特点，支持水下监测系统作用的 持续可靠发挥，保障领海权益不受侵犯。

\section{三、国内外海洋水下立体观测技术装备的发 展现状与趋势}

海洋水下立体观测系统主要涉及三方面：海洋 
观测平台、传感器、数据处理。鉴于数据（综合） 处理方面, 目前国内与国际存在较大差距, 加之专 业度较高、还需要更深层次的探究, 因此本文主要 围绕海洋水下固定观测平台（海洋水下观测网）、 水下移动观测平台、海洋传感器等装备展开论述。

\section{（一）海洋水下观测网}

海洋水下观测网计划由美国率先提出, 但因经 费问题而进展缓慢。1999 年 6 月, 加拿大率先建 成海王星海洋水下观测网; 通过复合缆将海底观 测点连成环路, 形成海底火山状态、地震海啸活动 的实时观测能力, 支持海洋与大气相互作用、气候 变化等方面的深入研究; 目前观测设备的数量超 过 138 个。进入 21 世纪, 各国积极建设海洋水下 观测网，如 2003 年日本建立的新型实时海洋水下 监测网（ARENA）、2004 年欧洲的海洋水下观测网 (ESONET)、2006 年澳大利亚的综合海洋观测系 统（IMOS）、2013 年印度的北印度洋海洋系泊浮标 (OMNI) 等。这些设施集防灾减灾、科学研究等多 类功能于一体, 观测传感器数量较多, 观测对象趋 于丰富，观测区域有效拓宽。

我国的海洋水下观测网 “十一五” 时期规划, “十二五” 时期建设, “十三五” 时期拓展；参与研 究和建设的单位有同济大学、浙江大学、中国海洋 大学、中国科学院声学研究所、中国船舶第七一五 研究所、亨通集团有限公司等 [7]。2009 年, 同济 大学牵头, 联合相关单位建成 “小倠山海底观测实 验站”，是我国第一个海底综合观测试验与示范系 统 [8]。2013 年, 中国科学院南海海洋研究所、沈 阳自动化研究所、声学研究所共同建设的 “三亚海 底观测示范系统” 投入运行 [9]。2014 年，浙江大 学建成了 “摘箬山岛海洋立体观测示范研究与试验 系统”。“十二五” 时期, 中国科学院声学研究所 牵头, 联合国内优势单位, 承研了国家 863 计划 重大项目 “海底观测网试验系统”, 重点攻关海底 观测网试验系统总体技术，突破了水下高压远程 供电、大深度高精度定位布放与回收、大深度水 下接驳盒与仪器、大深度高电压光电复合缆, 深 水无人遥控潜器 (ROV) 水下湿插拔等多项关键 技术，实现了深海海底观测网系统的建设与稳定运 行 [10]。2017 年, “海底科学观测网国家重大科技 基础设施” 项目建议书获批, 由同济大学牵头、中
国科学院声学研究所共建, 建设周期 5 年; 2018 年, “海底科学观测网国家重大科技基础设施” 项目可 行性研究报告获批。海底科学观测网是针对海底地 壳深部、海底界面、海水水体及海面开展高分辨率、 大范围、全天候、综合性、长期、连续、实时观测 的主要手段, 将为国家海洋安全、深海能源与资源 开发、环境监测、海洋灾害预警预报等研究提供支 撑。整体来看, 我国海洋水下观测网建设起步较晚, 目前仍未正式建成; 发展的新技术还需持续验证, 距离正式应用和推广尚有距离。

\section{（二）海洋水下移动观测平台}

海洋水下移动平台机动灵活, 如 ROV、载人 潜器 $(\mathrm{HOV}$ )、自主水下航行器 (AUV), 广泛应 用于海洋水下立体观测过程, 在海洋水下观测网的 安装和维修方面也发挥了重要作用。海洋水下观测 网为水下移动平台提供充电、信息传输中转等服务, 二者结合将发挥更大的作用。

\section{1. 无人遥控潜器}

20 世纪 60 年代, 国际上的许多海洋科学考察、 海洋资源探测活动, 较多利用遥控的无人水下观测 平台开展, 在较大水深中长时间工作的能力是主要 原因。ROV 在人工控制下, 在海洋深处、海底危 险区域执行各种指令与操作；除了开展观测基本任 务外, 还可通过机械手进行水下取样、安装、维护 等作业, 优势独特。ROV 技术成熟的国家有美国、 俄罗斯、日本、法国等，均具备研制 $6000 \mathrm{~m}$ 及以 下深度 ROV 的能力; 更大潜深的 ROV 还处于试验 阶段，尚未得到推广应用。

2020 年 6 月, 中国科学院沈阳自动化研究所研 制的 “海斗一号” ROV 完成了 $10907 \mathrm{~m}$ 的下潜深 度, 刷新了我国潜水器下潜深度及作业深度的记 录, 填补了万米作业型无人潜水器的空白。上海交 通大学研制的 “海马号” “海龙号” ROV 作业水深 分别为 $4500 \mathrm{~m} 、 6000 \mathrm{~m}$; 中国船舶第七一五研究所 研制的定型 ROV 作业水深为 $5000 \mathrm{~m}$ [11]。我国已 进入拥有 6000 米级 ROV 的国家行列。

\section{2. 载人深潜器}

HOV 最早出现在 20 世纪 60 年代, 由于航行 能力和作业能力有限、体积大、运输不方便, 未 能获得推广应用。美国 “阿尔文” HOV 于 1964 年 研制成功, 1966 年在地中海协助完成打捞工作, 
20 世纪 70 年代发现了海底热液喷口 [12]。“阿尔 文号” 最大潜深为 $4500 \mathrm{~m}$, 可让研究人员到达地 球上约 $2 / 3$ 的海底; 经过升级改进后, 最大潜深接 近 $6500 \mathrm{~m}$, 可覆盖 $98 \%$ 的海底面积。美国伍兹霍 尔海洋研究所 “深海挑战者号” 在马里亚纳海沟下 潜至 $10908 \mathrm{~m}$, 日本 “新海 6500 号” 最大潜深为 $6500 \mathrm{~m}$ 。

在我国, “蛟龙号” HOV 潜深为 7020 m; “深 海勇士号” HOV 国产化率达到 95\%, 核心国产设 备性能和技术状态稳定, 标志着我国大深度载人深 潜技术和装备制造取得重大进展。

\section{3. 自主式水下航行器}

AUV 造价低、安全性好、连续工作时间长, 可以自主设定航线、搭载多类水下传感器, 目标区 域的观测效果较好。国外 AUV 经过数十年的发展, 产品技术体系较为成熟, 功能性能基本稳定; 占据 国际市场主要份额的国家是美国、英国、挪威等, 市场上成熟的 AUV 产品类型超过 100 种。

在我国, 中国科学院沈阳自动化研究所等单位 牵头研制的 AUV 完成了水下 $6000 \mathrm{~m}$ 自主航行演 示, 仍处于研发测试阶段, 功能性能有待进一步验 证。未来 AUV 技术的发展方向是仿生性、多功能 性, 兼顾提升灵活性和续航性能。

\section{4. 水下滑翔器}

水下滑翔器依靠侧翼产生的水平动力在水下潜 行, 通过调节内部配重进行上浮和下潜; 搭载温盐 深 (CTD) 等多类传感器, 在水下大范围内持续搜 集环境参数, 成为海洋科考较为经济的水下观测工 具之一。美国最早开展水下滑翔器的研制, 目前拥 有最为成熟的技术; 法国、英国、日本等国家也很 早就开展各类水下滑翔器技术的研究。

中国科学院沈阳自动化研究所研制的 “海翼” 水下滑翔器, 工作水深为 300 7000 m、续航时间 达 $40 \mathrm{~d}$, 航程超过 $1000 \mathrm{~km}$ 。天津大学研制的 “海 燕” 水下滑翔器, 工作水深为 $3620 \mathrm{~m}$ 、续航时间 达 $30 \mathrm{~d}$, 航程超过 $1000 \mathrm{~km}$ 。相关产品的研制和 应用, 标志着我国基本掌握了水下滑翔器技术体 系 [10]。

\section{（三）海洋观测传感器}

随着海洋观测活动的不断发展, 在海洋水下、 海底、深海环境等长期连续观测需求的牵引下, 美
国、日本、德国等研制了多类海洋环境观测的新 型传感器, 如 CTD 传感器方面, 温度测量精度为 $\pm 0.001{ }^{\circ} \mathrm{C}$ 、电导率测量精度为 $\pm 0.003 \mathrm{mS} / \mathrm{cm}$ 、压 力测量精度为 $\pm 0.015 \% \mathrm{FS}$ 。

我国海洋观测传感器、仪器、测量系统研究起 步较晚, 得益于国家支持取得了较快进展: 一些技 术指标达到了国外同类产品的同期水平, 一些海洋 水下观测装备形成了系列产品, 一些水文仪器在大 洋考察、国际联合调查中得到应用。在国家 863 计 划、海洋公益性行业科研专项等渠道的支持下, 国 内研制了多型海洋调查、取样装备, 如自动气象站、 高精度 CTD 仪、海流剖面仪、磁力仪等; 发展的 深海取样装备有深海电视抓斗、多管取样器等 [13]。 海洋观测平台具有多种样式, 如浮标、潜标等被动 观测平台, 这些装备的研制和应用带动了海洋调查 观测、环境监测等专用传感器技术的进步。

\section{（四）海洋水下立体观测技术装备的发展趋势}

随着海洋水下观测需求的不断发展, 海洋观测 已经从海面转入水下、进而拓展到海底的全方位、 立体化观测阶段。这就要求海洋水下立体观测系统 涵盖多种观测平台, 如调查船、浮标浅标、水下潜 器、海床基观测站等; 通过多种观测平台的有机组 合, 形成水下全方位立体观测能力。多平台协同化 立体作业成为今后海洋观测的趋势, 观测范围也由 水面向水底拓展, 由近海向深远海延伸。在未来, 海洋水下观测平台将演进成为自适应、有机集成的 海洋水下观测平台阵列。

在深海环境和生态环境的长期连续观测需求牵 引下, 全海深绝对流速剖面仪、深海高精度海流计、 多电极盐度传感器、快速响应温度传感器、湍流剪 切传感器、多参数水质测量仪等海洋水下观测传感 器成为重点产品类别。伴随着海洋观测平台技术的 发展, 具有运动平台自动补偿功能的环境监测传感 器应运而生, 如适装于自治潜水器、遥控潜水器、 水下滑翔机、深海拖体等运动平台的温度、盐度、 湍流、 $\mathrm{pH}$ 、营养盐、溶解氧等传感器。在未来, 自 动补偿传感器改进应用于海洋水下观测平台阵列成 为重要发展趋势。

在未来的海洋水下观测体系中, 海洋传感器技 术朝着系列化、模块化、标准化、通用化方向发展, 海洋观测平台技术朝着多样化、多功能等方面发展。 
无人潜水器产业趋于成熟, 新型无人潜水器不断出 现, 海洋观测仪器与设备不断进步, 为海洋水下立 体观测技术装备构建提供了坚实支撑。此外, 得益 于数字信号处理器、大容量存拒器的进步, 海洋观 测仪器设备朝着小型化、多功能方向发展。

\section{四、我国海洋水下立体观测技术装备发展面 临的问题}

\section{（一）国产传感器与国际先进水平差距较大}

我国相关产业经过十多年发展, 在海洋观测平 台方面技术较为成熟, 但观测平台搭载的 CTD、光 学传感器等海洋观测核心传感器的进口比例依然较 高。除潮汐、海洋气象等测量仪器外, 大多数国产 海洋观测仪器设备的可靠性不高、产业化水平低。 入网观测仪器管理制度不够完善, 业务化海洋观测 仪器设备的研制、生产、应用、管理仍处于不协 调的 “亚健康” 状态; 业务化海洋观测的数据获 取能力、数据质量不高, 甚至在恶劣海况下难以 获得灾害环境预警所必需的基础数据。数据搜集、 后期维护改进等方面的技术难以自主掌握, 存在 着海洋观测活动在特殊时期遭遇设备断供、关键 信息泄露等问题。目前, 国内传感器研制大多处 于原理样机或工程样机阶段, 参与国际市场竞争 的实力薄弱; 一些核心技术仍待攻关, 传感器技 术的产业转化也需要通过实地监测、试验应用来 指导改进和提升。

\section{（二）海量数据与现实需求之间出现脱节}

海洋观测数据具有多重角色: 核心信息装备的 研发引擎, 海洋水下立体观测网的直接目的, 传输 网络的载体, 中心处理平台的依据, 海洋应用的基 础。经过长期发展, 国内的海洋遥感、岸基、水面、 水下等观测平台搜集了海量数据, 但目前相关数据 利用率、使用价值均不高; 从学术研究层面分析, 主要原因有: 反演信息欠精准, 信息提取知识欠科 学, 知识服务欠智能。获得海量数据之后, 应提高 数据使用的有效性, 切实为最终目标服务, 否则形 成 “数据孤岛” 而难以产生实际效能和投资回报。

\section{（三）国产传感器缺乏使用验证和必要改进} 我国海洋监测管理机构 “多头管理” 现象依然
存在, 导致研究力量分散、资源配置不合理, 制约 了重点工程实施成效, 不利于领域长远发展。在水 下立体观测网的信息处理和搜集方面, 目前服务对 象不够明确, 水下信息的流通范围较窄, 信息采集、 交换标准不一致, 数据整合度和利用率偏低, 社会 力量参与积极性不高, 不利于水下观测信息发挥应 用效能。以 863 计划海洋技术领域专项为例, 许多 国产传感器处于样机层面, 实地使用偏少, 也很难 继续进行必要的修正和改进; 许多国产传感器仅限 于试用或者 “沾水出数据” 即可, 未能进行产品化 并持续保持更新、改进、修正; 因后续的使用、改 进、实验等方面资源支持不足, 转化机制缺失, 在 研发和产业化之间出现了断层。

\section{五、海洋水下立体观测技术装备重点发展 方向}

海洋水下立体观测系统装备的建设, 除了有更 为成熟的海洋观测平台以外, 搭载在海洋平台上的 海洋传感器阵列成为海洋水下立体观测的关键。不 同类型的海洋水下观测平台, 在搭载海洋观测探测 仪器、海底取样等水下作业装备之后才能完整构成 装备体系, 也才具备充分发挥综合技术体系效能的 可能性。发展可供潜水器搭载的各类传感器、探测 装置、通用 / 专用作业工具, 是水下作业技术的基 本模式, 也是人类认识海洋、开发深水资源的重要 途径。

值得注意的是, 我国尽管在传统海洋观测传感 技术方面取得明显进展、逐步赶上国际先进水平, 但在新型传感器、特殊功能传感器方面依然面临 差距, 甚至出现了差距扩大的现象, 如高精准度 CTD 剖面仪、投弃式温深传感器、投弃式温盐深 传感器、声学多普勒流速剖面仪、相控阵声学海 流剖面仪等。应努力摆脱 “空壳建造、装备总装” 的现状, 改进海洋水下观测探测装备综合性能, 争取关键传感器的全面国产化; 跟踪移动平台、 组网观测等平台技术研究热点的同时, 及时谋划 并有效应对未来传感器小型化、低功耗、防污损 的挑战。

\section{（一）原位生物化学传感器}

物理海洋学传感器已经面世数十年, 国际上测 
量电导率、温度、深度的传感器也实现了高度成熟 和小型化, 但生物地球化学、生物海洋学类的观测 传感器进展明显滞后。除了监测可食用鱼类以外, 为实现海洋生态系统的可持续发展, 有必要理解和 掌握从浮游生物到鲸鱼在内的整个海洋生物链状 态。目前国内外在海洋生物学的监测技术方面都 不够成熟, 这是我国海洋生物传感器研究进步的 良好机遇。国际上生物监测逐步获得重视, 化学 测量新技术 (如硝酸盐、磷酸盐) 也在发展, 在 不远的将来有望成熟到可以持续利用的程度。我 国应与国际同步, 重视和发展相关类型的传感器, 如原位营养传感器、220 nm 紫外光谱测量仪、原 位自主硅酸盐电化学传感器、原位电化学硝酸盐 传感器等。

\section{（二）原位芯片海洋观测技术}

国际市场公开销售的基于试剂的化学、生物传 感器都没有采用原位芯片实验室 (LOC) 技术, 相 关技术仍处于少数国家 (如英国、日本)、少数实 验室的专利授权期。尽管微流体技术、LOC 技术 取得了新的进展, 但在海洋观测方面的应用潜力 仍待挖掘和明确。原位芯片海洋观测技术技术性 能高、具有潜在的低成本优势, 能够测量海洋水 下观测的多种参数, 非常适合填补目前海洋观测 能力的差距, 未来发展空间较大。原位芯片技术 的易用性提升极为重要, 在生物参数测量方面应 用潜力较大。

\section{（三）传感器主动防污技术}

生物污垢的积累会干扰传感器运行、减少流向 传感器的水流、影响平台机械运动、增加仪器质量 和阻力, 进而影响观测的准确性, 因此保护传感器 免受生物污染极为重要。传统处理方式主要有: 采 用防污涂料、涂层, 或选择防止生物污损的基质, 但对观测环境不友好; 配置擦拭器、通过设定不同 的擦拭频率来去除各种新沉积的污垢生物体; 采用 紫外线照射、淡水冲洗、纳米聚合物涂层等来防止 生物污垢沉积。在诸如东海等区域的高结垢环境中, 传感器需要综合采用多种方式来实施可靠保护。未 来传感器的发展, 应将生物附着性纳入评估内容, 根据结垢环境特征采用不同方式、不同频度的除垢 措施, 尤其需要关注主动擦拭器、电解氯化方案、
紫外线照射、纳米聚合物涂层等。

\section{六、对策建议}

\section{（一）支持海洋关键传感器的高效成果转化}

传感器是海洋观测最重要的构成部分, 性能 优劣直接反映海洋水下立体观测技术装备的水平高 低。国内存在着海洋传感器研制结题后因缺乏持续 必要支撑而难以实施成果转化的情况, 使得一些传 感器技术供应与市场需求结合不紧、产品研制缺乏 必要的试用与改进, 从而影响了前期研发投入的 产出和回报。海洋关键传感器尽管类别多样, 但 相关技术瓶颈具有共性, 这些问题应及时梳理并 集中解决。

建议开展行业顶层设计, 论证国家海洋传感器 综合规划, 争取事关海洋关键传感器研发与应用的 必要资源保障; 采取市场化方式、制定明确规则, 促进海洋关键传感器科技成果的高效转化; 鼓励原 始创新、推进应用创新, 重点支持国内需求迫切且 难以稳定进口渠道的传感器关键技术攻关和应用, 配套合理必要的政策和资源支持。

\section{（二）统筹建设海洋水下立体观测的大数据处理与 共享中心}

海洋观测耗资大、周期长、涉及多方, 多单位 甚至多国联合实施相关项目是常态。一方面, 围绕 海洋科学的重大问题, 基于通用的海洋观测平台, 实现不同学科背景、不同学术思想和不同层次的研 究力量协同, 旨在以学科交叉的形式高效推进领域 发展; 另一方面, 共享海洋观测平台可显著提高资 源利用效率, 更好促进观测装备及关键传感器的技 术进步与更新。随着观测技术装备的不断发展, 海 量数据不断积累, 数据处理、集成、产品化供应、 科学管理成为迫切任务。尽快落实海洋观测平台 共用、数据共享, 破解国内科研人员只能与国外 进行数据共享、一些国内产生的数据只能通过国 外途径来获取等不良现象, 扭转涉海数据自我封 闭、自我管理的局面, 是主管部门和科研人员共 同的责任。

建议将统筹建设海洋水下立体观测的大数据处 理与共享中心提上议事日程, 开展领域建设统筹规 划、构建数据共享制度; 充分利用并发挥好现有国 
家重大科技基础设施的作用，支持发展海洋水下立 体观测技术装备的核心关键技术，扎实提高核心装 备、关键传感器、观测装备的国产化水平，在海洋 环境监测与装备领域更快进入世界先进行列。

\section{（三）建立海洋水下立体观测技术装备国家公共试 验平台}

建立技术装备国家公共试验平台，有利于装备 测试与方案改进以提高性能, 可以持续培养海洋领 域的研究与技术人员, 积累装备与仪器的立项、研 制、改进经验, 加快工程应用进度。

建议开展海洋水下立体观测技术装备国家公共 试验平台建设，按照企业化、业务化、市场化方式 运作, 提供长期、连续、实时、多学科、同步、综 合的观测试验平台和基础设施功能; 建立资源共 享、要素完整、军民兼用的海上试验场, 为我国海 洋仪器与海洋模型的研发、检验和工程化应用提供 统一、优质、高效的服务。

\section{参考文献}

[1] 王芳. 构建我国海洋水下观测体系的思考 [N]. 中国海洋报, 2015-12-02(3)

Wang F. Thoughts on the construction of China's ocean underwater observation system [N]. China Ocean News, 2015-12-02(3).

[2] 吴润华. 水下遥控装置的现状及发展趋势 [J]. 海洋科技资料, 1980 (6): 44-57.

Wu R H. Present situation and development trend of underwater remote-control device [J]. Marine Science Bulletin, 1980 (6): 44-57.

[3] 汪品先. 从海底观察地球——地球系统的第三个观测平台 [J]. 自然杂志, 2007, 29(3): 125-130.

Wang P X. Seafloor observatories: The third platform for earth system observation [J]. Chinese Journal of Nature, 2007, 29(3): $125-130$.

[4] 吴自军, 周怀阳. 加拿大海底科学长期观测网的研究进展 [J]. 工 程研究, 2016, 8(2): 129-138.

Wu Z J, Zhou H Y. Research advances of ocean networks Canada
[J]. Journal of Engineering Studies, 2016, 8(2): 129-138.

[5] 惠绍棠. 水下自航式海洋观测平台技术发展研究 [J]. 海洋技术, 2001, 20(4): 11-17.

Hui S T. Research on technology development of autonomous underwater vehicle [J]. Ocean Technology, 2001, 20(4): 11-17.

[6] 石绥祥, 李占斌, 华彦宁, 等. 水下观测信息体系发展思考 [J]. 中 国工程科学, 2016, 18(2):61-65.

Shi S X, Li Z B, Hua Y N. Thoughts on the designs, applications, trends, and challenges of underwater observation information systems [J]. Strategic Study of CAE, 2016, 18(2): 61-65.

[7] 陈建冬, 张达, 王潇, 等. 海底观测网发展现状及趋势研究 [J]. 海 洋技术, 2019, 38(6): 95-103.

Chen J D, Zhang D, Wang X, et al. Research on the state-of-the-art and trends of seafloor observatory [J]. Ocean Technology, 2019, 38(6): 95-103.

[8] Xu H, Yu Y, Qin R. Tests and upgrades for the east China sea seafloor observatory [J]. Sea Technology, 2012 (9): 56-59.

[9] 殷建平. 南海首个海底观测示范系统在三亚成功建成 [EB/ OL]. (2013-05-13)[2020-08-30]. http://www.scsio.ac.cn/xwzx/ tpxw/201305/t20130513_3838180.html.

Yin J P. The first submarine observation demonstration system in the South China Sea was successfully built in Sanya [EB/ OL]. (2013-05-13)[2020-08-30]. http://www.scsio.ac.cn/xwzx/ tpxw/201305/t20130513_3838180.html.

[10] 常永国, 张飞, 郭永刚, 等. 南海深海海底观测网试验系统海底 动力观测数据集 [J]. 中国科学数据, 2019, 4(4): 48-55.

Chang Y G, Zhang F, Guo Y G, et al. The ocean dynamic datasets of seafloor observation network experiment system at the South China Sea [J]. China Scientific Data, 2019, 4(4): 48-55.

[11] 漆随平, 万运周. 海洋环境监测技术及仪器装备的发展现状与 趋势 [J]. 山东科学, 2019, 32(5): 21-30.

Qi S P, Li Y Z. A review of the development and current situation of marine environment observation technology and instruments [J]. Shandong Science, 2019, 32(5): 21-30.

[12] 朱心科, 金祥龙, 陶春辉, 等. 海洋探测技术与装备发展探讨 [J]. 机器人, 2013, 35(5): 376-384

Zhu X K, Jin X L, Tao C H, et al. Discussion on development of ocean exploration technologies and equipments [J]. Robot, 2013, 35(5): 376-384.

[13] 牟健. 我国海洋调查装备技术的发展 [J]. 海洋开发与管理, 2016, 33(10): 78-82.

Mou J. The development of China's marine survey equipment technology [J]. Ocean Development and Management, 2016, 33(10): 78-82. 